\title{
Anabases
}

ANABASES Traditions et réceptions de l'Antiquité

21 | 2015

Varia

\section{Francisco DE VITORIA, De la loi}

\section{Karine Wurtz}

\section{(2) OpenEdition \\ Journals}

Édition électronique

URL : http://journals.openedition.org/anabases/5367

DOI : 10.4000/anabases.5367

ISSN : 2256-9421

\section{Éditeur}

E.R.A.S.M.E.

\section{Édition imprimée}

Date de publication : 1 avril 2015

Pagination : 288-290

ISSN : 1774-4296

\section{Référence électronique}

Karine Wurtz, «Francisco dE VITORIA, De la loi », Anabases [En ligne], 21 | 2015, mis en ligne le 01 avril 2015, consulté le 22 septembre 2020. URL : http://journals.openedition.org/anabases/5367 ; DOI : https://doi.org/10.4000/anabases.5367

Ce document a été généré automatiquement le 22 septembre 2020.

(c) Anabases 


\title{
Francisco DE VITORIA, De la loi
}

\author{
Karine Wurtz
}

\section{RÉFÉRENCE}

Francisco DE VITORIA, De la loi, Commentaire, introduction et notes de Gaëlle

Demelemestre, Paris, Éditions du Cerf, 2013, 180 p.

25 euros / ISBN 978-2-204-10003-8

1 On connaît surtout de Vitoria ses grandes « leçons » annuelles, notamment celles sur les indiens et sur le droit de guerre. On connaît moins l'enseignement régulier, son analyse approfondie de la pensée de Thomas d'Aquin, mise en perspectives avec les débats d'époque. C'est ce manque que la traduction de Gaëlle Demelemestre vient combler aujourd'hui, en nous permettant de découvrir le cours donné par Vitoria pendant l'année universitaire 1533-1534 sur le fameux Traité des lois de Thomas d'Aquin, correspondant aux questions 90 à 108 de la Somme Théologique, Ia-IIae.

2 Après une introduction de Blandine Kriegel rappelant tout ce que la modernité doit à Vitoria, l'ouvrage nous propose une introduction éclairante de Gaëlle Demelemestre. L'auteur commence par rappeler la signification de la loi chez Thomas d'Aquin, pour ensuite s'arrêter sur les nominalistes (Pierre Auriol, Durand de Saint-Pourçain, Duns Scott, Guillaume d'Occam...) qui se sont constitués autour de la critique de l'ontologie thomasienne. L'introduction revient pour finir sur les thèses luthériennes. Nous avons ainsi un panorama utile des différentes pensées convoquées par Vitoria pour commenter le Traité des lois.

Gaëlle Demelemestre rappelle que chez Thomas d'Aquin, Dieu est avant tout Sagesse. La loi est donc l'expression de la raison de Dieu, elle traverse le réel comme puissance créatrice et organisatrice selon un modèle hiérarchique : au sommet se trouve la loi divine, de celle-ci participe la loi naturelle dans une moindre perfection, puis de même pour la loi humaine, chacune étant parfaitement adaptée au domaine normatif qu'elle régit. Tout le système est tendu par un postulat : la possibilité pour l'homme d'accéder au bonheur suprême et à la vie éternelle. Les lois humaines, dont le but est la concorde 
entre les hommes, doivent permettre le développement et l'exercice de la charité. Ainsi, bien qu'elles soient circonstancielles, les lois positives expriment le juste dont elles participent en tant que lois. De plus, la loi naturelle est accessible à l'homme pour s'imposer à lui comme une évidence. Susceptible de s'en écarter par l'exercice de sa liberté, l'homme possède alors en lui les principes de la responsabilité. Enfin, la loi divine dépasse l'intelligence humaine et reste inconnaissable. L'auteur rappelle ainsi que la théorie de la loi se double chez Thomas d'Aquin d'un enseignement pratique : l'ordre inhérent au réel doit servir de principe normatif de l'action.

4 L'auteur rappelle ensuite la critique nominaliste : si pour Thomas d'Aquin, Dieu est avant tout Sagesse, pour les nominalistes, il est avant tout Volonté. La loi peut donc être retirée ou modifiée selon son arbitraire. Les conséquences sont multiples : le droit revient relatif, de même que la justice. Le réel lui-même n'est plus qu'un contingent muet. Par conséquent, la loi positive devient la seule norme du bien et du mal. Dès lors, la bonne ou la mauvaise conscience n'a plus de signification, la grâce n'est pas liée à une réforme intérieure. Dans la conception nominaliste, la loi a avant tout un sens pénal, elle est un instrument extérieur permettant aux hommes de coexister. Le respect de l'obligation sera ainsi le fondement de la moralité.

La dernière opposition majeure aux théories thomasiennes que Vitoria convoque est tenue par Luther. Pour lui, le péché originel a tellement perverti l'homme qu'un abîme infranchissable s'est creusé entre la justice divine et le monde. La puissance rationnelle ou les inclinations naturelles ne peuvent lui permettre de distinguer le bien du mal, il est incapable d'avancer seul vers la sainteté et ne peut qu'espérer que la foi lui sera offerte. L'homme ne peut donc que s'humilier. Dans cette pensée, la loi naturelle n'a plus de sens, la loi ne peut concerner que l'espace politique. Elle n'est qu'une force extérieure, qui interdit et punit, sans effet sur les consciences.

6 Cette introduction, à la fois dense et claire, se termine par un résumé des enseignements délivrés par Vitoria au fil de son commentaire : il renforce la loi naturelle, il réaffirme les deux fonctions de la loi : ordonner les relations humaines et contraindre.

7 L'ouvrage présente ensuite le texte du cours de Vitoria, dans une excellente traduction réalisée par Gaëlle Demelemestre. Il consiste en vingt-huit " questions ", chacune traversée par plusieurs articles répondant à chaque fois à une interrogation. Pour chacune, Vitoria expose le texte thomasien, propose son propre commentaire, convoque les objections possibles chez les opposants, évalue ces objections sans aucun passage en force, puis donne sa conclusion. Après avoir discuté de l'essence de la loi (90), de sa diversité (91) et de ses effets (92), le texte s'arrête sur la loi éternelle (93), la loi naturelle (94) et la loi humaine (95-97). Viennent ensuite des questions consacrées à la loi ancienne (98-100), les préceptes cérémoniels (101-103), les préceptes judiciaires (104-105) et enfin la loi nouvelle (106-108). C'est ainsi l'occasion de traiter des interrogations comme " la loi est-elle toujours orientée au bien commun ? ", « la coutume peut-elle obtenir force de loi ? " ou encore "la loi naturelle peut-elle changer?"

Le commentaire de Vitoria est précieux à plus d'un titre : d'une part, il expose avec beaucoup de rigueur et d'exhaustivité les thèses thomasiennes. D'autre part, bien que sa faveur aille à Thomas d'Aquin, Vitoria leur oppose les meilleurs arguments des thèses nominalistes sans esquiver la difficulté. Son étude poussée des thèses nominalistes auprès de Petrus Crockaert à Paris ne peut qu'ajouter à l'intérêt de son 
évaluation. Cette démarche, alliant rigueur et grande honnêteté intellectuelle, autorise un réel débat, qui non seulement permet l'exposition d'un auteur fondamental de la pensée politique moderne, mais aussi permet l'exposition d'un panorama de la connaissance et des positions intellectuelles sur la question de la loi au Xvi ${ }^{\mathrm{e}}$ siècle.

\section{AUTEURS}

\section{KARINE WURTZ}

Université de Paris 1 Panthéon-Sorbonne

Karine.wurtz@gmail.com 